\title{
UNIVERSITY OF CALIFORNIA.
}

AGRICULTURAL EXPERIMENT STATION.

BERKELEY, CAL.

E. W. HilgARD, Director. BULLETIN No. 115.

\section{Remedies for Insects and Fungi.}

DECEMBER, 1896. 



\section{UNIVERSITY OF CALIFORNIA \\ AGRICULTURAL EXPERIMENT STATION}

\section{(Correction and Supplement to Bulletin II5.)}

This sheet is published in response to numerous inquiries for formulas not included in Bulietin I I 5, recently issued by this Station, and for the purpose of correcting quite an important typographical error in the above-mentioned bulletin.

\section{W. WOODWORTH.}

\section{CORRECTION.}

O11 page I2, lines 12 and I 3 , the formula for the Lime, Salt, and Sulphur Mixture should read: "The proportions are about 6 pounds of linie, 2 of salt, and 3 of sulphur, for ro (not 6o) gallons."

\section{SUPPLEMENT.}

Professor Hilgard's Sulphid of Potash Wash.--This has proven so useful for removing moss from trees that a number have requested it. The formula is as follows: Caustic soda $\left(99^{\circ}\right)$, I pound; commercial potash, I pound; sulphur, 3 pounds. Boil together one hour. Dissolve 20 pounds whale oil soap $\left(80^{\circ}\right)$ in hot water; add, and boil one half-hour. Dilute to roo gallons. Apply hot.

Professor Pierce's Bordcaux Mixture.-This is the modification of the Bordeaux mixture with which Professor Pierce had such good results against the curl-leaf of the peach. It contains double the amount of lime generally recommended. The formula is as follows: 5 pounds of bluestone, Io pounds of lime, for 45 gallons. Prepare in the same manner as other Bordeaux mixtures. For curl-leaf apply from one to three weeks before buds open.

Paris Green and Bordeaux Mixture.-We neglected to state in the Bulletin that when it is desirable to use these two washes at the same time, as for codlin moth and scab, the green may be stirred into the Bordeanx mixture at the usual proportion, i.e., I pound to 200 gallons. 
Digitized by the Internet Archive in 2012 with funding from University of California, Davis Libraries 


\section{REMEDIES FOR INSECTS AND FUNGI.}

By C. W. WOODWORTH.

It is very important that every one who has the care of crops, whether of garden, orchard, or field, should have a clear idea of the remedies available for preventing the injuries due to insects and fungi. Not only must one know how to make and apply the remedies, but it is equally essential that he should fully understand when to apply and when not to apply. It is safe to say that, even in California, where this matter has been agitated for so many years, in only a very small fraction of the cases where injury might be prevented is the proper treatment made. On the other hand, it may also be said that when a treatment is made it is often of no effect, and a waste of time and money. Careful observation of the practices in this State in reference to treating insects and fungi makes it appear that fully half of what it now costs to treat our crops is wasted.

Mistakes Made.-The chief mistakes made in applying remedies are the following:

First-Applications are often made when there is absolutely no need for treatment-when there is nothing to destroy nor to prevent;

Second-Applications are often wrongly timed; either applied before there is any chance to produce good results, or after the injury has all been done;

Third-Often the wrong kind of an application is chosen for the particular trouble, so that no results can follow;

Fourth-Applications are often made for troubles that are incurable by any known practical method;

Fifth-Expensive methods are employed when cheaper ones will accomplish the results, both as regards the material used and the labor employed.

There is no reason why these might not to a great degree be avoided. Mistakes of judgment will always cause loss in this as in any other operation, but there is no reason why these mistakes of ignorance should be allowed to amount to half the cost of such applications. In the following pages we will attempt to present, in a brief compass, the more important points to be considered in the treatment of plants. But it should be distinctly recognized that local experience and experiment is the only real guide for successful practice. Especially in this State, where our conditions are so wonderfully diversified, is it impossible to formulate detailed programs or calendars of operations which will be of general value.

Any one with ordinary intelïigence, however, who is willing to observe and think, has no excuse for falling into the mistakes indicated above. 


\section{CLASSES OF PESTS.}

Almost all the serious pests that attack plants are either insects or fungi. These we may classify, according to their way of attacking the plant, into a number of groups, as follows:

1. Root-feeding insects, which attack the roots of plants; they may devour the roots, suck the sap, or cause swellings to form; and the same insect may even attack the plant above ground as well as on the roots.

2. Boring insects, which live within the plant and mostly attack the stem or trunk, but may also bore into the larger roots on the one hand and into the interior of leaves and fruit on the other.

3. Sap-sucking insects, which attack the upper parts of the plant, puncturing the leaves and stems to obtain their food. These resemble the forms that suck the sap from the roots, but the latter are a different and much more difficult economic problem.

4. Defoliating insects, which eat up the leaves and other green parts of plants.

5. External fungi, growing over and living upon the exposed parts of the leaves and stems of plants, and only sending feeding suckers into the plant.

6. Local fungi, which enter the plant, but remain at the point where they found an entrance, and only spread by the spores, which correspond to seed, being carried to other parts of the leaf or plant and there growing into the plant again.

7. Penetrating fungi, which have the power of passing from one part of a plant to another by boring their way right through the plant.

Each of these classes of pests must be treated in a different way, and usually the remedy for one would be entirely useless if applied for a pest of another class.

\section{INSECTS.}

Root-Feeding Insects.-Fortunately the number of root-feeding insects is not large, for there is no application known, that is practical for general use, by which they can be treated. It is not that the insects are hard to kill, but that the nature of the soil is such that it is very difficult to reach them. The best of the known processes - the use of carbon bisulphid-is not certainly effectual, unless used in such quantity as to destroy at the same time the roots of the plants; and thus is practically useless, except for disinfecting soils. The very exhaustive and unsuccessful experiments made with the phylloxera, especially in France, make it appear doubtful if anything will ever be found in the way of treatment for root insects.

The solution of the problem for trees and vines seems to be along the line of the use of resistant roots.

For annual crops, the most promising methods at present known are crop rotation, starvation, and trapping.

The crop rotation method depends for its success upon the fact that most of the injurious root-feeding insects or worms are decidedly more numerous in a certain field in certain years, and especially after certain crops. By planting only those crops that are the least injured by the attacks in the years following these crops, the injury can, to some degree, be avoided. Local experience will show the particular system of rota- 
tion which will give the best results with the insects most abundant in that particular region.

The starvation method is by clean fallowing the land, and is not particularly useful after a crop like potatoes, where there is left considerable living vegetable matter in the soil. It is the most thorough method if everything is kept off the ground, and can be used to advantage in connection with trapping.

The trapping method consists of providing food for the insects which has first been treated with a strong dose of poison. It is especially useful in gardens where root-feeding insects are often a very serious pest. Any green food will do for traps. Alfalfa is commonly used. It is scattered around in small piles and may be partly covered with soil to prevent it from drying out too fast.

- Boring Insects.-About the only thing that can be done for boring insects is to prevent, by some means, their entrance into the plant. This may be done by mechanical means, by covering the threatened parts with something which will either prevent the egg-laying or form a barrier to the young insect. Thus, the use of cylinders of wire mosquito net, an inch or two larger than the trunk of the trees, is an effectual bar against the borer that attacks the butts of peach trees. Borers may also be prevented entrance by coating the part of the plant, liable to attack, with a poisonous substance, which will be eaten by the young borer as it attempts to burrow into the plant. Such a coating for the trunks of trees is a paint composed of glue made green with Paris green. For green parts of plants, especially against fruit insects, Paris green is applied in water and the application must be repeated often enough to keep the parts well poisoned.

After the insects have entered the plant, about the only thing remaining to be done is to dig them out one by one, though for some living very near the surface it is said that scalding hot water applied freely to the trunk will destroy the insect without injury to the tree.

It is doubtful, however, if by either of these means enough injury is not directly or indirectly done to the plant to more than equal the good attained.

Sap-Sucking Insects.-In this category are included some of the easiest as well as some of the most difficult insects to destroy, but they are all capable of successiul treatment, so far as we know. It may often be, however, that the cost of a treatment which is efrective will be so great as to be prohibitive. This is true generally of field crops, where the cost per acre for treatment may often be more than the saving that can ensue from the application. The insects of this class are not affected by poisons like Paris green, because they get their food by inserting their beak into the plant; nothing, therefore, on the surface of the leaves will have any particular effect.

There have been many attempts at injecting some substance into the plant which will poison the sap, but the cells of the plant are, if anything, more delicate than the insect, so that the plant is always killed first.

The only feasible plan, therefore, is to spray on them some caustic or oily substance, or to envelop them with some poisonous gas.

Plant-lice are ordinarily very easy to kill, but protected insects, like 
the scale insects, or very active ones, like the so-called grape thrips, are much more difficult to deal with. The latter are probably best treated by causing them to leap or fly against a "hopper doser" of some form. A hopper doser consists of a surface of tin, or other material, covered with tar, printer's ink, or some other sticky substance, which will hold captive the insects touching it.

Defoliating Insects.-The insects eating the leaves of plants are, as a rule, the easiest insects to destroy, and at the least expense, because the leaves may be covered with relatively cheap poisonous substances; and as the insects eat the leaf, they will also consume the poison and be killed. Only the more valuable field crops can be treated economically, however, for even as cheap a process as this is too expensive for most of the staple crops.

When insects are excessively abundant, as in the case of attacks of locust swarms and invasions of army worms, all known applications become useless, because with their great numbers everything green is devoured before any one insect can have taken enough poison to kill it.

Insects of the defoliating class can also be killed by the same remedies used for sap-sucking forms; but with those remedies, as is true of the insects of the former class, the insect must actually be touched by the remedy to be affected by it.

\section{FUNGI.}

External Fungi.-The molds or mildews occurring on the leaves of plants in this State belong almost exclusively to this class of fungi.

One can easily, with the aid of a small microscope, see the threads of the fungus. All parts of the plant above ground are affected, but the young leaves and the ends of stems are most readily attacked.

They may be destroyed and the spread of the fungus stopped by the use of the vapor of sulphur.

Local Fungi.-Most of the rusts and spot fungi may be classed under this head. 'They live within the plant, and so cannot be destroyed after they once gain an entrance. A cure, therefore, is impossible, and attention must be turned to preventing their entrance. The spores are produced very abundantly and quickly in most species, so that, if treatment is not given soon after the first appearance of the trouble, it will be seen to spread with great rapidity and involve a much larger part of the plant.

The theory of treatment is to keep the plant covered with some substance which will prevent the germination or sprouting of the spores, thus preventing the fungus from entering a new part of the plant. We now know a number of very effectual substances which will produce this result.

Penetrating Fungi.-This is the most difficult group of fungi we have to deal with. Grain smuts, blackberry rust, and curl-leaf of the peach are examples. In annual plants the infection usually occurs at about the time that the seed sprouts, so that the destruction of the spores that may be on the seed is often sufficient to insure practical immunity.

In perennials, the only thing usually to be done is the removal and destruction of the infested plants. 
Some diseases of trees due to these fungi may possibly be controlled by preventing the spread of the disease (by spores), by the use of the remedies that are so effectual against the local fungi, and by vigorous pruning and removing the new growth, in which the disease is chiefly carried over from one season to another. This is, at least at present, the most promising line of experiment in these cases.

The experience in some parts of this State with curl-leaf, where it seems to be entirely controllable, would seem to indicate that the disease is not, in every region, to be properly classed with these penetrating fungi.

Further study may show that other fungi may, in certain seasons or in certain regions, be a local and in others a penetrating fungus, and so in one place be easily controlled, and in another not at all controllable without the destruction of the plants affected.

\section{REMEDIES.}

There are a great many substances that may be used successfully against insects and fungi; but we recommend only a small list, selected because of their effectiveness and cheapness. Remedies are applied as a dry powder, as a gas, or as a fluid, spray or wash; the great majority being in the latter form.

Powders. - The most common way of applying powders is the "pepperbox" method, in which the material is carried in a vessel provided with perforations, through which it sifts as the vessel is shaken over the plant. A modification of this, much used in the cotton fields of the Southern States, consists of bags of the material suspended from the ends of a pole long enough to reach from one row of cotton to the next. This is carried by a man riding on a mule, and the jar causes the powder to sift through. Cloth is chosen for this purpose which is fine enough to allow only the right quantity to be distributed. A third method, much used in this country, is a blowing device, which is very satisfactory for field use, and does very rapid work. Only three remedies are recommended to be used in the dry form, and these have a rather limited use.

Air-Slaked Lime.-This is the powder resulting from the exposure of ordinary lime to the action of the air for some time. It is only recommended as a remedy against insects which have a slimy coating over the body. For these it is a cheap and effectual remedy. It is not as cheap, however, as Paris green, and is particularly recommended where the latter is objectionable because of its poisonous nature.

For true slugs, which are not insects, but have a similar slimy coat, it is the best remedy we know of ; but it must be applied in the evening or early morning, while the animals are on the plants; and may have to be repeated two or three nights in succession to kill all. Except in gardens, it may not pay to make more than the one application, which will, if rightly timed, destroy most of the slugs.

Sulphur.-This is a widely used remedy for the mildew, which is so common on plants in this State. The powder is usually applied by the pepper-box method. It only becomes effectual as the heat of the sun 
vaporizes it; the field thus treated smells strongly of the sulphur during the warmer part of the day, when the vapor is being produced. On wet, cloudy days, when the mildew is growing and spreading rapidly, the sulphur is inert, but the first bright day makes it effective. It may be possible to artificially vaporize the sulphur on a large scale for use in such weather; but no attempt has yet been carefully made, except in greenhouses, and then with the best of results. The vapor of sulphur must be used, and not the gas produced by burning, which is very injurious to foliage.

Sulphur has been found to be a successful remedy for the so-called red spiders, also called yellow mite-animals somewhat related to the true insects; fairly good results are also reported in its use against the thrips. For these purposes the sulphur is used in the same way as for the mildew.

Paris Green or London Purple.*-These two arsenical poisons are occasionally used in a dry form, but chiefly suspended in water as a spray. They are used for the same purposes in either form. A common practice is to dilute the poison with flour, dust, or other powder, so that it can be more easily and evenly distributed. If not so diluted, care must be taken not to apply it so thickly in places as to endanger the foliage.

\section{Gas Treatments.}

Gases have the property of diffusing themselves with great rapidity, so that when applied in a closed space every part of that space will in a short time contain some of the gas. Thus, no other method of killing insects is calculated to be as thorough in its work. In an open space this property of diffusing destroys, to a great extent, its utility. Sulphur applied as a powder, as has already been stated, is not effective until it becomes a vapor; and then the dissipation is not as much as with most gases, because of the weight of sulphur vapor and the fact that it is applied over whole fields at once.

The impracticability of inclosing most cultivated plants, and the cost of the treatment, both in time and chemicals, makes the method useful to only a very limited extent. We recommend but two gases.

Carbon Bisulphid.-This substance is not available for plants in active growth, and is chiefly useful for stored products, such as seeds and grains. It can be used for disinfecting soils and ridding other articles of insects.

In disinfecting soils an injector is used. A number of forms of the latter are on the market in Europe, where phylloxera eradication has required them. It is doubtful if this method of soil disinfection would be profitable in this country for the destruction of any insect.

For destroying insects in seeds or grain, carbon bisulphid is a very cheap and satisfactory means. The bin or box containing the seeds to be disinfected should be tight, at least at the sides and below.

A dish is placed on top of the material to be treated, and a quantity of the carbon bisulphid poured in. This evaporates rapidly, and the vapor, being heavier than air, sinks down into the lower part of the bin.

*For a general account of their action, see under sprays. 
The top should be covered also, in order that the vapor may remain a long time in the grain.

Very rarely will bins be perfectly tight, so that the exact amount necessary cannot be stated. Usually the estimate is made at one pound to the ton of grain, which is sufficient for a fairly tight bin.

Grapevine cuttings are most successfully disinfected in a similar way, by placing a saucerfull of the bisulphid on the cuttings in a tight box, and leaving for forty minutes.

The yellow jacket, our common wasp, which is so injurious to fruit, and which makes its nest in the ground, is easily killed by this substance. The nest is located, and about dark, when the wasps are all in, about an ounce of carbon bisulphid is poured down the hole and a handful of earth thrown over it to keep the vapor in. By morning all will be found to be dead. Ants' nests can be destroyed in the same way.

Carbon bisulphid is also one of the best remedies for gophers. If used in the fall, after the rains have begun (so that the ground is not too porous), an ounce poured over a rag and stuffed into a hole and covered with earth will destroy the inmates.

Hydrocyanic Acid Gas.--This is practically the only gas which is strong enough to kill the insects on a tree with safety to the leaves, and within a time short enough to make its use practical on a large scale. Its work is very effectual and satisfactory, but is very expensive, and, therefore, is only available for the more profitable trees, such as the citrus varieties. It may also be used for disinfecting. The gas is extremely poisonous, and, sometimes, for reasons not entirely understood, is very injurious to the foliage; but the injury is almost all prevented by its use at night.

The process is to cover the tree with a tent of sail-cloth, oiled to make it tight, and in a vessel beneath the tent the chemicals which make the gas are placed. After half an hour the tent is removed and put over the next tree. The chemicals needed for every 100 cubic feet are $\frac{1}{3}$ ounce of potassium cyanide, $\frac{2}{3}$ ounce of water, and $\frac{1}{3}$ ounce of sulphuric acid.

The common practice in this State is to use it decidedly stronger for small trees and weaker for the largest trees. In the smaller trees it is safe to use it stronger, as the small amount of gas used is very quickly diffused. When it is produced in large quantity, as is necessary for a large tree, some of the gas, scarcely at all mixed with air, may come in contact with the leaves and injure them. The practice probably arose, in part, from some tables which have been published widely in this State, where the contents of the tent was calculated by multiplying the height of the tree by its breadth. The practice is not justifiable, and if the weaker amount is successful there is no reason for not using the same proportion on smaller trees.

There are many kinds of tents used, and many plans for changing them from tree to tree, the details of which we cannot give here. The tent is usually of an octangular shape, and is furnished with rings for hoisting. The largest tents require a derrick for lifting them into place. 
The following are the amounts of cyanide of potash to be used for trees of varying heights:

Height of Tree.

Amount of Cyanide of Potash.

\begin{tabular}{c|c} 
If as broad as & If $2 / 3$ as broad as \\
high (Navel & high (Seedling \\
Orauges, etc.) & Oranges, etc.)
\end{tabular}

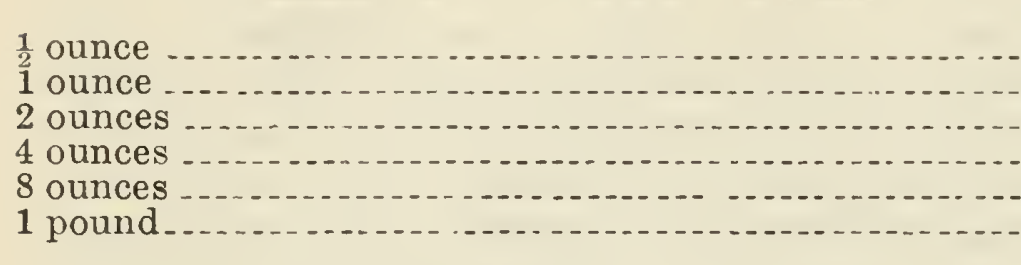

6 feet.

8 feet.

10 feet.

12 feet.

15 feet.

19 feet.

The most important remedies for plant pests are applied as a spray or wash. By the word wash one would naturally understand a more copious application than a spray, but in ordinary usage the terms are identical.

For very low plants an outfit working on the principle of a sprinklingcan will do; but for better and more economical work, and work on higher plants, some form of force-pump and spray nozzle is necessary.

There are many forms of pumps on the market that are good for the purpose. A good spray pump should maintain a fairly constant and sufficient pressure, and its valves and general construction should be simple and its parts easily replaceable.

The nozzle should, for most spraying work, be such as to break up the stream into a fine mist; but for scale insects one giving a stream of considerable force is desirable. The shape of the spray is usually either conical or fan-shaped, and each has its particular advantages for a particular class of work.

The construction of the nozzle should be such as to permit of easy, quick, and thorough cleaning. A few forms designed to clean themselves automatically, work well.

In most spraying the object is to get the largest possible proportion of the spray to remain on the leaf or stem, and to have it well distributed. This is best accomplished by covering the leaf with minute globules like dew. As soon as they run together and drip from the leaf the distribution is not perfect, and there is actually less left on the leaves.

When the nozzle is held as far from the plant as the stream will carry, the full effect of the nozzle is obtained in the breaking up of the stream into mist, and so can produce most perfectly this dew-like condition.

For scale insects the object is somewhat different. The plan for these is to thoroughly wet the surface of the bark and the edges of the scale, to insure the penetration of the wash beneath the scale, and thus to kill the old scale or the eggs and young hidden beneath. This thorough wetting is secured by holding the nozzle close to the plant and applying a great deal of the wash with a high pressure. Washes are sometimes applied hot, and when the nozzle is held close to the plant the spray will penetrate better, and for this reason do better work. 
Heat is to be recommended as a remedy only when it can be absolutely controlled.

Grain Smut.-The method of treating seed wheat and oats for smut is as follows:

For wheat, soak fifteen minutes in water at $132^{\circ} \mathrm{F}$; for oats, ten minutes. The grain should first be placed in water at, say, $115^{\circ}$, to partly warm it up. See that the grain is all wetted and do not treat too much at a time, or it will be impossible to keep the water at the proper temperature. Have boiling and cold water at hand, so as to add to keep the temperature just right. It must not go above $135^{\circ}$, or it may injure the seed; nor below $130^{\circ}$, or it will not be effective. After treating, dip in cold water to cool, and spread out to dry.

Bordeaux Mixture.-This is the most used and the most satisfactory of the known washes for prevention of fungous attacks. When sprayed upon the plant it prevents the development of the spores of any fungi upon the surface covered; and it is thus a perfect preventive of all the fungi which gain an entrance to the plant in this way. To be effective it must be kept continuously on the plant that is to be protected. A single spraying will last from two weeks to a month, or even longer if the conditions are favorable. With our dry summers one application will often be found enough for some kinds of fungi.

Late spring rains may make additional sprayings necessary; and some fungi may, in some localities, require the repeated sprayings necessary in a more humid climate. Local experience will have to determine these points.

Bordeaux mixture is made of equal parts of bluestone (copper sulphate) and lime. Some formulas give 6 parts of bluestone to 4 parts of lime, and will do if the lime is good. Slake the lime, and dissolve the bluestone separately. Both should be cold when they are mixed, and the resultant mixture will be a beautiful blue wash. If mixed hot, a black compound (copper oxide) is produced, which reduces the value of the wash.

As to the amount of water, we recommend, for ordinary spraying, 1 pound of each of the above to 10 gallons of mixture; and for winter use, 1 pound of each for 4 gallons.

Ammoniacal Copper Carbonate.-This solution is very effectual, but is not as lasting as the Bordeaux mixture. It is a perfect solution, and therefore has no tendency to clog the nozzle (as is the case with the Bordeaux mixture), and can be used on fruit near the picking season, and on ornamental plants, which would be disfigured with the lime wash.

The usual way of making this wash is to dissolve copper carbonate in ammonia, and then dilute. If the carbonate is not fully dissolved before the water is added it cannot be further dissolved, and not only is the carbonate wasted, but the fluid will not be up to standard strength. It is well, therefore, to give the ammonia ample time to act, say over night, before adding the water. The proportions are 1 ounce of the copper carbonate in 10 ounces of ammonia for every 10 gallons of spray.

Another way of producing the mixture is to mix solutions of bluestone and sal soda, dissolving the carbonate of copper thus produced in ammonia, and diluting with water. The bluestone and the sal soda 
should be mixed in about the proportion of 3 to 4, after having been dissolved separately in a small quantity of warm water, and cooled before mixing.

Three ounces of bluestone with 4 of sal soda and 20 of ammonia is sufficient for 20 gallons of spray.

Lime, Salt, and Sulphur Mixture.-This wash is useful both as an insecticide and a fungicide, but only for winter use on deciduous trees.

It is usually made by boiling the sulphur for one hour and a half with about one fourth of the lime, in a covered kettle, with enough water to cover well; then the rest of the lime and the salt are added, and the boiling continued half an hour longer.

The proportions are about 6 pounds of lime, 2 of salt, and 3 of sulphur, for 60 gallons.

Resin Soap.-The cheapest insecticide which kills by contact is resin soap. It is for scale insects, and so has good penetrating power. Like all insecticides killing by contact, the effect of the spray is soon gone, and it only kills the insects which are wet with its spray. It is generally applied warm.

In making the soap, the ingredients are placed in a closed kettle with enough water to cover, and are boiled for two hours, when all will be united into a soap. In diluting, only a little water should be added at a time, and stirred in; or, better, hot water should be used, because of the danger of chilling the soap and causing it to harden, when it is almost impossible to dissolve it again.

The proportions are 8 pounds of resin, 2 pounds of standard caustic soda, and 1 pint of fish oil for 40 gallons, for use on trees in foliage; and the same for 25 to 30 gallons for winter use.

Kerosene.-There has recently been placed on the market devices for mixing kerosene with water in the pump as it is being applied; and with very good results. Full directions as to proportions accompany the device, and we will here only discuss the precautions to be observed. These are, chiefly, the use of a nozzle capable of producing a very fine mist; and applying the spray so that the drops do not run together, i.e., holding the nozzle as far as possible from the plant, and not applying too much.

Pure kerosene has great penetrating power, perhaps the greatest of all the insecticides, and with the above precautions can be safely used.

Kerosene Emulsion.-While not as cheap as resin soap, kerosene emulsion is easier to make; and, in small quantities, or where the conveniences for boiling are not at hand, is the most satisfactory remedy. Like resin soap, this spray only kills by contact.

The ordinary form of the emulsion is something of an art to properly manufacture. The ingredients are 2 parts of kerosene to 1 of sour milk, or of strong soap solution. The latter must be made boiling-hot and added to the kerosene, and the whole pumped through a spray nozzle for fifteen minutes. After pumping a few minutes the whole mass will become beautifully creamy and apparently perfectly emulsified; but if a little is placed in water it will be seen that some of the kerosene separates out and rises to the surface. When the emulsion is 
perfect there will be no separation when diluted. If the soap is of poor quality or the water is hard, more soap must be used; and, on the other hand, if both are good not as much is needed as recommended below, which is intended to suit the average condition.

The proportions are 3 ounces of soap in 3 pints of water or sour milk, and 3 quarts of kerosene for 10 gallons of emulsion for scale insects, or for 15 gallons for plant-lice.

Another formula, which is easier made but more expensive, and so only recommended for use on a small scale, consists of using eight times as much soap. When so made, the creamy mixture described above, obtained within five minutes, is a permanent emulsion.

Paris Green and London Purple.-These poisons kill only those insects that eat the leaves covered by them, and are useless against sucking insects like plant-lice and scales. They are also useless against overwhelming numbers of insects, such as swarms of grasshoppers, which are able to eat up the plant before getting enough poison to kill them.

Arsenic is the active principle in both these poisons, and is about equally strong in both. Paris green is copper arsenite, and London purple an arsenite of lime containing an aniline residue, to which the purple color is due. The latter is a finer powder and remains better suspended in water, but the Paris green is, as a rule, safer to apply. Both poisons, if applied too strong, will burn the foliage, and both require constant stirring, or the material will settle to the bottom, and so not be uniformly distributed. For the best results, the poison should not be allowed to drip from the tree, and the finer the spray the better.

These poisons do not as a rule remain any great length of time on the plant, but must be renewed every two or three weeks, or while the danger of insect attack exists. In mixing the poison it is well to first make a paste with a little water and then dilute, as otherwise it is difficult to wet the leaves with it. The amount used is about one pound to two hundred gallons of water. 


\section{THE TABLE.}

The following table is intended to give, in a glance and in small compass, the ingredients, proportions, and method of preparation of these various washes. For convenience they are calculated in per cents for metric calculations; while the amounts necessary for 5 and 40 gallons respectively (as representing an oil-can and barrel) are given in the common weights and measures. The table should be tacked up and preserved for future reference: 


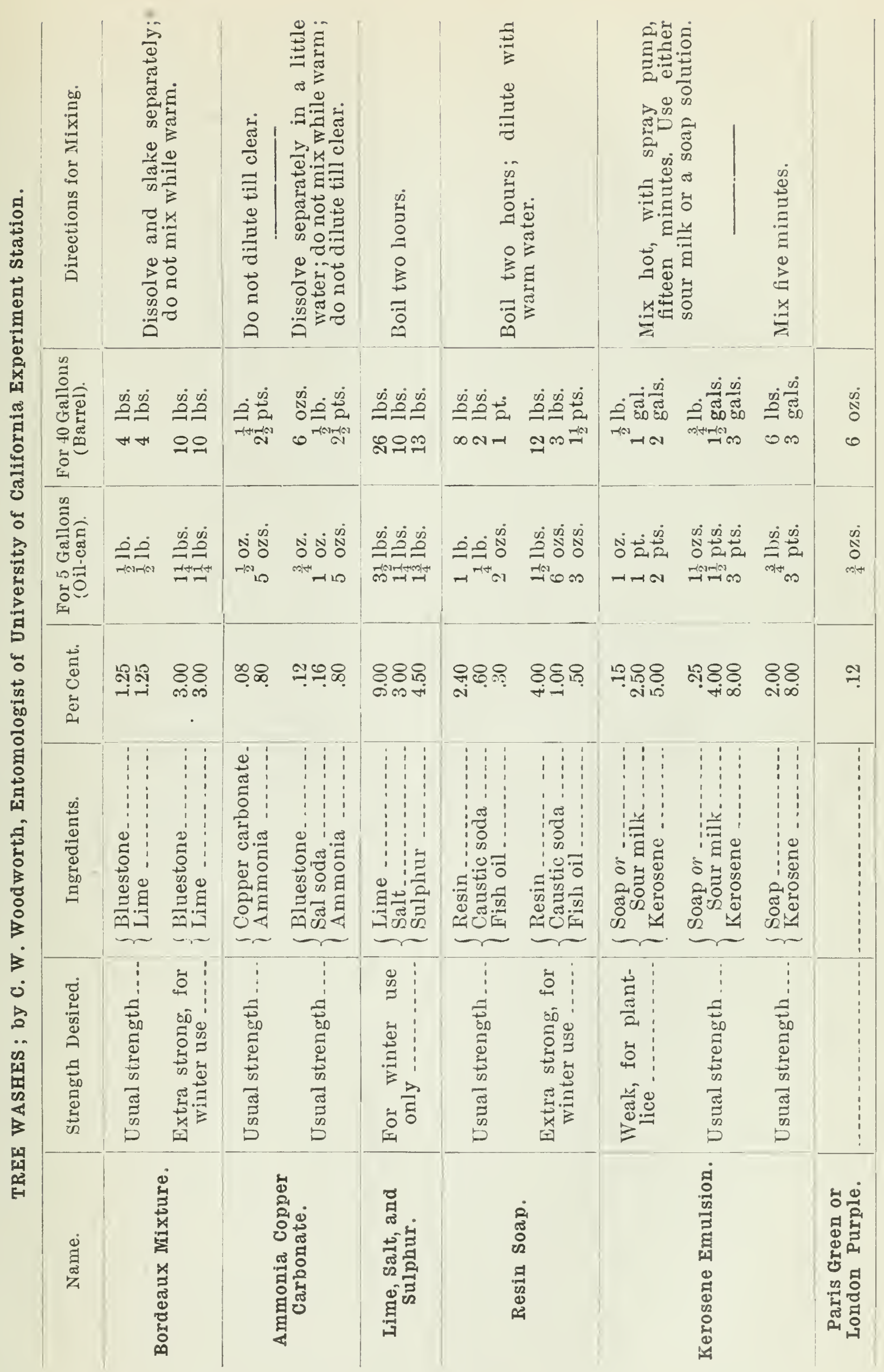


